\title{
Microwave Analysis for Two-Dimensional C-V and Noise Model of AlGaN/GaN MODFET
}

\author{
Ramnish Kumar, ${ }^{1}$ Sandeep K. Arya, ${ }^{1}$ and Anil Ahlawat ${ }^{2}$ \\ ${ }^{1}$ Department of ECE, Guru Jambheshwar University of Science and Technology, Teaching Block 7, Hisar, Haryana 125001, India \\ ${ }^{2}$ Department of CSE, Krishna Institute of Engineering and Technology, Ghaziabad, Uttar Pradesh 201206, India
}

Correspondence should be addressed to Ramnish Kumar; contactram1@rediffmail.com

Received 12 May 2014; Revised 5 August 2014; Accepted 6 August 2014; Published 10 September 2014

Academic Editor: Baibiao Huang

Copyright (C) 2014 Ramnish Kumar et al. This is an open access article distributed under the Creative Commons Attribution License, which permits unrestricted use, distribution, and reproduction in any medium, provided the original work is properly cited.

\begin{abstract}
A new two-dimensional analytical model for the capacitance-voltage and noise characteristics of a AlGaN/GaN MODFET is developed. The two-dimensional electron gas density is calculated as a function of device dimensions. The model includes the spontaneous and polarization effects. The contribution of various capacitances to the performance of the device is shown. The model further predicts the transconductance, drain conductance, and frequency of operation. A high transconductance of $160 \mathrm{mS} / \mathrm{mm}$ and a cut-off frequency of $11.6 \mathrm{GHz}$ are obtained for a device of $50 \mathrm{~nm}$ gate length. The effect of gate length on the gate length behaviour of the noise coefficients $P, R$, and $C$ is also studied. The effect of parasitic source and gate resistance has also been studied to evaluate the minimum noise figure. The excellent agreement with the previously simulated results confirms the validity of the proposed model to optimize the device performance at high frequencies.
\end{abstract}

\section{Introduction}

HEMT is very useful in designing Microwave Monolithic Integrated Circuits (MMIC) and also for designing the circuits at higher frequency range of operation. The superior performance of HEMTs over conventional MESFET structures has been demonstrated in both analog and digital ICs. In recent years, AlGaN/GaN pHEMTs (Pseudomorphic High Electron Mobility Transistors) have emerged as a very promising device for high frequency, high power density, and high temperature applications $[1,2]$. The pHEMTs on $\mathrm{GaN}$ are rapidly replacing conventional MESFET technology in military and commercial applications. AlGaN/GaN pHEMTs have emerged strongly as attractive candidate for high power, high temperature, and high speed applications in the microwave regions. The presence of strong spontaneous and piezoelectric polarization fields between AlGaN and $\mathrm{GaN}$ in addition to the large band-gap energy, high saturation velocity, large breakdown fields, large conduction band discontinuities, and high thermal stability are some of the characteristic features of the device which leads to outstanding performance on GaN-based HEMTs. Capacitancevoltage characteristics are widely used as a diagnostic tool for semiconductor. The $\mathrm{C}-\mathrm{V}$ characteristics also provide more information about the nonlinearity of the device for the large signal models [3-6]. Low noise HEMTs are promising devices for millimetre wave due to their excellent high frequency and low noise performances. Because of the high carrier density and high breakdown fields, AlGaN/GaN pHEMTs have low noise performance over conventional GaAs-based HEMTs. The HEMT appears to be an ideal device for low noise application $[7,8]$. The earliest study of HEMTs consisted of a one-dimensional analytical approach, which is not sufficient to describe the behaviour of these devices in the submicrometer regime. A number of analytical models [9-11] have been developed so far to characterize the 2-DEG sheet carrier concentration. But these models do not explain the behaviour of the device in the subthreshold region. Therefore to provide a better physical understanding of the device operation, an analytical approach to model the 2-DEG density of AlGaN/GaN pHEMTs is highly desirable.

In the present paper, a new analytical model for an AlGaN/GaN pHEMT is realised by solving the 2D Poisson's equation in the depleted $\mathrm{AlGaN}$ region to characterize the $\mathrm{dc}$ performance of the device considering the effect of parasitic 


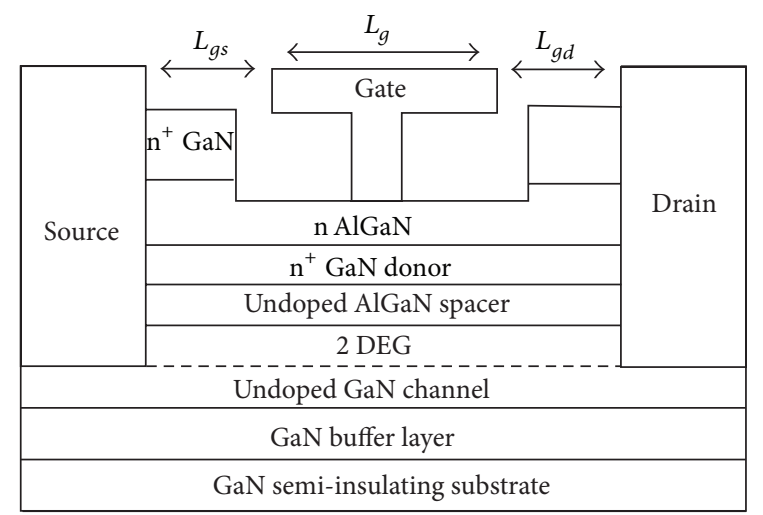

(a)

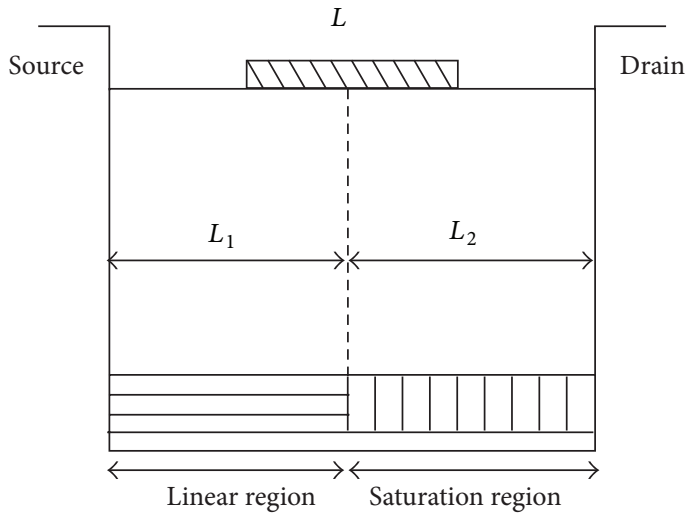

(b)

Figure 1: (a) Schematic of AlGaN/GaN MODFET. (b) Diagram for showing linear and saturation region used for 2D analysis.

resistances. A detailed analysis of various polarization effects associated with it is to be performed. The model includes the effects of various fringing capacitances due to the small geometry of the device. These device capacitances are then used to evaluate the microwave performance of the model. The model evaluates the transconductance, cut-off frequency, transit time, minimum noise figure, and noise coefficients of the device. Such models are helpful as they provide good insight into the physical operation of the device. The device capacitances, both gate, drain, and gate, source, play a vital role in determining the microwave performance of the pHEMT. The results so-obtained are compared with the previously published experimental and simulated results, which prove the validity of the model.

\section{Model Formulation}

2.1. Charge-Control Model. The basic structure of AlGaN/ GaN pHEMT [12] is as shown in Figure 1.

The device structure consists of GaN quantum well channel separated by undoped AlGaN spacer layer from thick n-type AlGaN supply layer. The function of the undoped spacer layer is to reduce impurity scattering. The structure of the device is shown with drain and source electrodes as ohmic contacts. HEMTs or AlGaN/GaN MODFETs are high performance devices for their potential use at higher frequencies because of the GaN as substrate, which offers thermal stability and high electron velocity. The buffer layer is epitaxially grown on the substrate. Many pHEMTs contain a super lattice structure to further inhibit substrate conduction. A super lattice structure is a periodic arrangement of undoped epitaxial layers used to realize a thicker epitaxial layer of a given property. The most important point about the channel layer in the HEMT and pHEMT devices is the twodimensional electron gas (2DEG) that results from the bandgap difference between AlGaN and GaN.

Other typical models of pseudomorphic HEMT (pHEMT) are rapidly replacing conventional MESFET technology in military and commercial applications requiring low noise figures and high gain particularly at millimeter wave frequencies. The application of pHEMTs for high efficiency power amplifications is gaining popularity.

The 2DEG sheet charge density can be obtained by simplifying and solving Poisson's equation [5] as

$$
n_{s}(m, x)=\frac{\varepsilon(m)}{q \cdot D}\left(V_{\mathrm{gs}}-V_{c}(x)-V_{\mathrm{th}}(m)\right),
$$

where $D$ is the separation between the gate and the channel, $q$ is the electronic charge, $m$ is the Al mole fraction, $V_{\mathrm{gs}}$ is the applied gate-source voltage, $\varepsilon(m)$ is the AlGaN/GaN dielectric constant, and $V_{c}(x)$ is the channel potential at $x$ due to the drain voltage. $V_{\text {th }}$ is the threshold voltage and is given as

$$
\begin{aligned}
V_{\mathrm{th}}(m)= & \phi_{m}\left(T_{0}\right)+m\left\lfloor\varepsilon_{\mathrm{gap}}^{\mathrm{AIGaN}}(T)-\varepsilon_{\mathrm{gap}}^{\mathrm{AIGaN}}\left(T_{0}\right)\right\rfloor \\
& -\frac{\Delta E_{g}}{2}-\frac{E T}{2} \ln \left[\frac{N_{c}^{\mathrm{AIGaN}} N_{V}^{\mathrm{GaN}}}{N_{V}^{\mathrm{AIGaN}} N_{C}^{\mathrm{GaN}}}\right]+\Delta E_{\mathrm{exc}}-\Delta E_{\mathrm{im}} \\
& +\Delta E_{b}-\frac{q N_{d} d_{d}^{2}}{2 \varepsilon(m)}-\frac{q N_{b}}{c_{b}}+\frac{E_{f}(m)}{q}
\end{aligned}
$$

where $\phi_{m}\left(T_{0}\right)$ is the time-dependent Schottky barrier height, $\Delta E_{\text {im }}$ is the dielectric image force, $\Delta E_{b}$ is the interface dipole bond energy, $N_{V}$ and $N_{C}$ are the electron concentration in the valence and conduction band, respectively, $\Delta E_{c}(m)$ is conduction-band discontinuity of $\mathrm{AlGaN} / \mathrm{GaN}$ interface, $N_{d}$ is the doping concentration of the AlGaN barrier, $\sigma(m)$ is the net polarization induced sheet charge density at the AlGaN/GaN interface, $\Delta E_{\text {exc }}$ is the electron exchangecorrelation energy, and $E_{f}(m)$ is the Fermi potential. 
The total polarization induced sheet charge density is given [3] as

$$
\begin{aligned}
\sigma(m)= & P_{\mathrm{sp}}\left(A l_{m} G a_{1-m} N\right)-P_{\mathrm{sp}}(\mathrm{GaN}) \\
& +P_{\mathrm{PZ}}\left(A l_{m} G a_{1-m} N\right)-P_{\mathrm{PZ}}(\mathrm{GaN}),
\end{aligned}
$$

where $P_{\mathrm{SP}}$ and $P_{\mathrm{PZ}}$ are the spontaneous and piezoelectric polarization of $\mathrm{AlGaN}$ and $\mathrm{GaN}$ layers, respectively. The total amount of polarization induced sheet charge density for $\mathrm{AlGaN} / \mathrm{GaN}$ heterostructure field effect transistor is obtained as

$$
\begin{aligned}
& |\sigma(m)| \\
& \quad=\left|P_{\text {sp }}\left(A l_{m} G a_{1-m} N\right)-P_{\text {sp }}(\mathrm{GaN})+P_{\mathrm{PZ}}\left(A l_{m} G a_{1-m} N\right)\right| .
\end{aligned}
$$

In the above expression, it has been assumed that the GaN layer is fully relaxed. This is a reasonable assumption since the thickness of GaN layer is much larger than that of strained AlGaN layer. Thus, $P_{\mathrm{PZ}}(\mathrm{GaN}) \approx 0$.

And

$$
\begin{aligned}
& P_{\mathrm{PZ}}(\left.A l_{m} G a_{1-m} N\right) \\
&=2\left(\frac{a(0)-a(m)}{a(m)}\right) \\
& \times\left(e_{31}(m)-e_{33}(m) \frac{c_{13}(m)}{c_{33}(m)}+P_{\mathrm{sp}}(m)\right. \\
&\left.-P_{\mathrm{sp}}(0)\right) \mathrm{C} / \mathrm{m}^{2} \\
& P_{\mathrm{SP}}\left(A l_{m} G a_{1-m} N\right)=-0.052 m-0.029 \mathrm{c} / \mathrm{m}^{2} \\
& P_{\mathrm{SP}}(\mathrm{GaN})=-0.029 \mathrm{c} / \mathrm{m}^{2} .
\end{aligned}
$$

The piezoelectric polarization induced charge density depends upon $\mathrm{Al}$ mole fraction which is given as

$$
\begin{aligned}
& P_{\mathrm{PZ}}\left(A l_{m} G a_{1-m} N\right) \\
& =\left\{\begin{array}{l}
\left(\frac{2(a(0)-a(m))}{a(m)}\right)\left(e_{31}(m)-\frac{e_{33}(m) C_{13}(m)}{C_{33}(m)}\right) \\
\text { for } 0 \leq m \leq 0.38 \\
\left(\begin{array}{c}
2(2.33-3.5 m) \\
a(m) /(a(0)-a(m))
\end{array}\right) \\
\times\left(e_{31}(m)-\frac{e_{33}(m) C_{13}(m)}{C_{33}(m)}\right) \\
\text { for } 0.38 \leq m \leq 0.67 \\
0, \quad \text { for } 0.67 \leq m \leq 1,
\end{array}\right.
\end{aligned}
$$

where $a(m)$ and $a(0)$ are lattice constants, $e_{31}(m)$ and $e_{33}(m)$ are piezoelectric constants, and $c_{13}(m)$ and $c_{33}(m)$ are elastic constants, respectively.
For a given aluminum mole fraction, there is a certain critical thickness of barrier layer. Equation (6) shows that the device remains completely strained for $\mathrm{Al}$ content up to $38 \%$ and fully relaxed for $\mathrm{Al}$ content greater than $67 \%$ for thickness range of $200-400 \AA$.

2.2. Current-Voltage Characteristics. The drain-source current in the channel is obtained from the current density equation and is given [13] as

$$
\begin{aligned}
& I_{\mathrm{ds}}(m, x) \\
& \quad=z q \mu(x, m)\left(n_{s}(m, x) \frac{d v_{c}(x)}{d x}+\frac{K_{B} T}{q} \frac{d n_{s}(m, x)}{d x}\right),
\end{aligned}
$$

where $z$ is the gate width, $K_{B}$ is Boltzmann constant, $T$ is temperature, and $\mu(x)$ is the field dependent electron mobility and is given [2] as

$$
\mu(m)=\frac{\mu_{0}(m)}{1+\left(\left(\mu_{0}(m) E_{c}-V_{\text {sat }}\right) / E_{c} V_{\text {sat }}\right)\left(d v_{c x} / d x\right)} .
$$

By substituting (1) and (8) in (7) and on integrating using boundary conditions

$$
\begin{gathered}
\left.v_{c}(x)\right|_{x=0}=I_{\mathrm{ds}} R_{s}, \\
\left.v_{c}(x)\right|_{x=L}=V_{\mathrm{ds}}-I_{\mathrm{ds}}\left(R_{s}+R_{d}\right),
\end{gathered}
$$

where $R_{s}$ and $R_{d}$ are the parasitic source and drain resistances, respectively.

The drain to source current in the channel is given as

$$
I_{\mathrm{ds}}=\frac{-\alpha_{1}+\sqrt{\alpha_{1}^{2}-4 \alpha_{2} \alpha_{3}}}{2 \alpha_{2}},
$$

where

$$
\begin{aligned}
& \alpha_{1}=E_{1}\left(2 R_{s}+R_{d}\right)-\frac{E_{2} R_{d}}{2}\left(R_{d}+2 R_{s}\right), \\
& \alpha_{2}=-\left[L+E_{1} V_{\mathrm{ds}}+E_{2}\left(V_{\mathrm{gs}}-V_{\mathrm{th}}-\frac{K_{B} T}{q}\right)\right. \\
& \left.\times\left(2 R_{s}+R_{d}\right)-E_{2} V_{\mathrm{ds}}\left(R_{s}+R_{d}\right)\right], \\
& \alpha_{3}=E_{2}\left(V_{\mathrm{gs}}-V_{\mathrm{th}}-\frac{K_{B} T}{q}\right) V_{\mathrm{ds}}-\frac{E_{2}}{2} V_{\mathrm{ds}}^{2} .
\end{aligned}
$$


The drain saturation voltage $V_{\mathrm{dsat}}$ is obtained as

$$
V_{\mathrm{dsat}}=\frac{-\delta_{1}+\sqrt{\delta_{1}^{2}-4 \delta_{2} \delta_{3}}}{2 \delta_{2}}
$$

where

$$
\begin{aligned}
\delta_{1}= & \frac{\mu_{0} Z \varepsilon(m) E_{c}}{d}\left[\frac{\alpha_{1} \mu_{0} z \varepsilon(m) E_{c}}{d}+E_{1}-E_{2}\left(R_{s}+R_{d}\right)\right] \\
& -\frac{E_{2}}{E}, \\
\delta_{2}= & \frac{\mu_{0} Z \varepsilon(m) E_{c}}{d}\left[\frac{-2 Q \alpha_{1} \mu_{0} z \varepsilon(m) E_{c}}{d}+E_{1} Q\right. \\
& \left.+E_{2} Q, \quad Q\left(R_{s}+R_{d}\right)+L+E_{2} Q\left(2 R_{s}+R_{d}\right)\right] \\
\delta_{3}= & \frac{Q \mu_{0} Z \varepsilon(m) E_{c}}{d} \\
& \times\left[\frac{\alpha_{1} \mu_{0} z \varepsilon(m) E_{2} Q}{d}-L-\frac{E_{2}\left(2 R_{S}+R_{d}\right)}{d}\right]
\end{aligned}
$$

with

$$
Q=V_{\mathrm{gs}}-V_{\mathrm{th}}-\frac{K_{B} T}{q}
$$

The detailed analysis for (10) and (12) is reported in [6].

2.3. Capacitance-Voltage Characteristics. The equation for channel current in terms of position dependent sheet carrier concentration and electron velocity is given as [10]

$$
I_{\mathrm{ds}}=Z q n_{s}(x) v_{d}(x)
$$

where $Z$ is the gate width, $q$ is the electronic charge, $n_{s}(x)$ is the electron concentration in the channel, and $V_{d}(x)$ is the electron drift velocity and is given as

$$
V_{d}(x)=\frac{\mu_{0} E(x)}{1+\left(1 / E_{3}\right) E(x)}
$$

where

$$
E_{3}=\frac{E_{c} V_{\text {sat }}}{\mu_{0} E_{c}-V_{\text {sat }}}, \quad E(x)=\frac{d V_{c}(x)}{d x}
$$

and $\mu_{0}$ is the low field mobility

Total channel charge is given as

$$
Q=\int_{0}^{t} I_{\mathrm{ds}} d t=\int_{0}^{L} \frac{I_{\mathrm{ds}} d x}{v_{d}(x)}
$$

Rearranging (15) and using (1), we get

$$
\frac{I_{\mathrm{ds}}}{v_{d}(x)}=\frac{Z \varepsilon(m)}{D}\left\{V_{\mathrm{gs}}-V_{\mathrm{th}}(m)-V_{c}(x)\right\}
$$

Integrating (19) from 0 to $L$,

$$
\int_{0}^{L} \frac{I_{\mathrm{ds}}}{v_{d}(x)} d x=\frac{Z \varepsilon(m)}{D} \int_{0}^{L}\left\{V_{\mathrm{gs}}-V_{\mathrm{th}}(m)-V_{c}(x)\right\} d x
$$

using [10]

$$
d x=\frac{Z q n_{s} \mu_{0}-\left(I_{\mathrm{ds}} / E_{3}\right)}{I_{\mathrm{ds}}} d V_{c}(x) .
$$

By substituting (21) into (20) and using boundary conditions in (9), the total channel charge is obtained as

$$
\begin{aligned}
Q=-\frac{A_{1}}{3 I_{\mathrm{ds}}}\left[B-\left\{V_{\mathrm{ds}}-I_{\mathrm{ds}} R\right\}\right]^{3}-\left[B-I_{\mathrm{ds}} R_{s}\right]^{3} \\
-A_{2}\left[B\left\{V_{\mathrm{ds}}-I_{\mathrm{ds}} R\right\}-B I_{\mathrm{ds}} R_{s}-\frac{1}{2}\left\{V_{\mathrm{ds}}-1_{\mathrm{ds}} R\right\}^{2}\right. \\
\left.+\frac{1}{2} I_{\mathrm{ds}}^{2} R_{s}^{2}\right],
\end{aligned}
$$

where

$$
\begin{aligned}
A_{1} & =\frac{z \varepsilon(m)^{2} z \mu_{0}}{D^{2}}, & B & =V_{\mathrm{gs}}-V_{\mathrm{th}}, \\
R & =R_{s}+R_{d}, & A_{2} & =\frac{Z \varepsilon(m)}{E_{3} D} .
\end{aligned}
$$

2.3.1. Gate-Drain Capacitance. It is also called feedback capacitance. It is defined as the rate of change of charge on the gate electrode with respect to the drain bias when the source and the gate potentials are kept constant:

$$
\begin{aligned}
C_{\mathrm{gd}}= & \frac{\partial Q}{\partial V_{\mathrm{ds}}} \\
= & \frac{-A_{1}}{3 I_{\mathrm{ds}}}\left[3\left\{B-\left(V_{\mathrm{ds}}-1_{\mathrm{ds}} R\right)\right\}^{2}\left\{1-g_{d} R\right\}\right] \\
& +\left[\left\{B-\left(V_{\mathrm{ds}}-I_{\mathrm{ds}} R\right)\right\}\right]^{3}\left[\frac{A_{1}}{3 I_{\mathrm{ds}}^{2}} g_{d}\right] \\
& +\frac{A_{1}}{I_{\mathrm{ds}}} \cdot\left(B-I_{\mathrm{ds}} R_{s}\right)^{2}\left(-g_{d} R_{s}\right)
\end{aligned}
$$




$$
\begin{aligned}
+(B- & \left.I_{\mathrm{ds}} R_{s}\right)^{3}\left(\frac{-A_{1}}{3 I_{\mathrm{ds}}^{2}} g_{d}\right) \\
-A_{2} & {\left[B\left(1-g_{d} R\right)-B R_{s} g_{d}-\left(V d s-I_{\mathrm{ds}} R\right)\left(1-g_{d} R\right)\right.} \\
& \left.+I_{\mathrm{ds}} R_{s}^{2} g_{d}\right] .
\end{aligned}
$$

2.3.2. Gate-Source Capacitance. The gate-source capacitance $\left(C_{\mathrm{gs}}\right)$ is defined as the change in total charge with the change in gate voltage. It is mainly due to the $2 \mathrm{DEG}$ in the normal operating region and is given as

$$
\begin{aligned}
C_{\mathrm{gs}}= & \frac{\partial Q}{\partial V_{\mathrm{gs}}} \\
= & \frac{-A_{1}}{3 I_{\mathrm{ds}}}\left[3\left\{B-\left(V_{\mathrm{ds}}-I_{\mathrm{ds}} R\right)\right\}^{2}\left\{1-g_{m} R\right\}\right] \\
& +\left\{B-\left(V_{\mathrm{ds}}-I_{\mathrm{ds}} R\right)\right\}^{3} \frac{A_{1}}{3 I_{\mathrm{ds}}^{2}} g_{m} \\
& +\frac{A_{1}}{I_{\mathrm{ds}}} \cdot\left(B-I_{\mathrm{ds}} R_{s}\right)^{2}\left(1-g_{m} R_{s}\right) \\
+ & \left(B-I_{\mathrm{ds}} R_{s}\right)^{3}\left(\frac{-A_{1}}{3 I_{\mathrm{ds}}^{2}} g_{m}\right) \\
& -A_{2}\left[\left\{B\left(-g_{m} R\right)+\left(V_{\mathrm{ds}}-I_{\mathrm{ds}} R\right)\right\}-\left\{B g_{m} R_{s}+I_{\mathrm{ds}} R_{s}\right\}\right. \\
& \left.\quad-\left(V_{\mathrm{ds}}-I_{\mathrm{ds}} R\right)\left(-g_{m} R\right)+I_{\mathrm{ds}} R_{s}^{2} g_{m}\right] .
\end{aligned}
$$

\section{Small-Signal Parameters}

3.1. Drain/Output Conductance. It is an important microwave parameter that determines the maximum voltage gain attainable from a device. The drain conductance of the $\mathrm{AlGaN} / \mathrm{GaN}$ pHEMT is evaluated as

$$
\begin{gathered}
g_{d}(m)=\left.\frac{\partial I_{\mathrm{ds}}(m)}{\partial V_{\mathrm{ds}}}\right|_{V_{\mathrm{gs}}}, \\
g_{d}(m) \\
=\frac{1}{2 \alpha_{1}}\left[-E_{1}+E_{2}\left(R_{d}+R_{s}\right)+\frac{1}{2 \sqrt{\alpha_{1}^{2}-4 \alpha_{2} \alpha_{3}}}\right. \\
\left.\times\left\{2 \alpha_{2}\left(-E_{1}+E_{2}\left(R_{d}+R_{s}\right)\right)-4 \alpha_{1} E_{2}\left(Q-V_{\mathrm{ds}}\right)\right\}\right] .
\end{gathered}
$$

3.2. Transconductance. It is the most important parameter for optimization of FET high frequency behaviour. The major part of the gain mechanism is embodied in the active channel transconductance, which is evaluated as

$$
\begin{aligned}
& g_{m}(m)=\left.\frac{\partial I_{\mathrm{ds}}(m)}{\partial V_{\mathrm{gs}}}\right|_{V_{\mathrm{ds}}}, \\
& g_{m}(m)=\frac{1}{2 \alpha_{1}}\left[-E_{2}\left(2 R_{s}+R_{d}\right)+\frac{1}{2 \sqrt{\alpha_{1}^{2}-4 \alpha_{2} \alpha_{3}}}\right. \\
& \left.\times\left\{2 \alpha_{2}\left(-E_{2}\left(2 R_{s}+R_{d}\right)\right)-4 \alpha_{1} E_{2} V_{\mathrm{ds}}\right\}\right] .
\end{aligned}
$$

3.3. Cut-Off Frequency $\left(f_{t}\right)$. The unity gain cut-off frequency is an important figure of merit of the MODFET performance at microwave frequencies. The high velocity of electrons in the channel at the drain side results in a high transconductance. The cut-off frequency is given as the ratio of the transconductance to the sum of capacitances (both $C_{\mathrm{gs}}$ and $C_{\text {gd }}$ ); that is,

$$
f_{t}=\frac{g_{m}}{2 \pi\left(C_{\mathrm{gs}}+C_{\mathrm{gd}}\right)} .
$$

And by substituting the value of $g_{m}$, which has already been derived in (27) and $C_{\mathrm{gd}}$ and $C_{\mathrm{gs}}$ from (24) and (25), respectively, the cut-off frequency can be evaluated. The cutoff frequency $f_{t}$ determines the ultimate speed of a switching device.

3.4. Transit Time. The transit time effect is the result of a finite time being required for carriers to traverse from source to drain. Smaller transit times are desirable to attain a high frequency response from a device. The transit time for the $\mathrm{AlGaN} / \mathrm{GaN}$ pHEMT is evaluated as

$$
T_{t}(m)=\frac{g_{m}}{2 \pi f_{t}(m)}
$$

By substituting (28) in (29), transit time can be obtained.

\section{Power Added Efficiency}

In certain applications such as mobile and satellite communications [14], DC power is at a premium. In modern communication system and radar applications, the RF power amplifier may be the subsystem that consumes most of the DC power. For this reason, the efficiency of the RF power amplifier can be critical to the design and performance of the overall system. 
The basic efficiency is expressed as

$$
\eta=\frac{P_{\text {out }}}{P_{\mathrm{dc}}} \times 100 \%
$$

When input RF power is included in the efficiency calculations, the modified expression is referred to as Power Added Efficiency (PAE) and is expressed [15] as

$$
\begin{aligned}
\mathrm{PAE} & =\frac{P_{\mathrm{out}}-P_{\mathrm{in}}}{P_{\mathrm{dc}}}=\frac{P_{\mathrm{out}}}{P_{\mathrm{dc}}}\left(1-\frac{P_{\text {in }}}{P_{\mathrm{out}}}\right) \\
& =\frac{P_{\text {out }}}{I_{\mathrm{ds}} V_{\mathrm{ds}}}\left(1-\frac{1}{G}\right) \times 100 \%,
\end{aligned}
$$

where $G$ is the power gain and is given as

$$
G=\frac{P_{\text {out }}}{P_{\text {in }}}
$$

\section{Noise Analysis}

In the present work, the noise is produced in the ohmic section of the channel in region I termed as thermal or Johnson noise. And in region II, the noise is produced in the velocity saturated section of the channel region termed as diffusion noise. The analytic expressions for the mean square values $\overline{i_{g}^{2}}$ and $\overline{i_{d}^{2}}$ are obtained in this section.

5.1. Drain Noise Source. The drain voltage fluctuation due to an elementary noise produced at $x$ is obtained as

$$
\overline{\Delta v_{d 1}^{2}}=\frac{4 k T_{0} \Delta f}{I_{d}} \cosh ^{2}\left(\frac{\pi L_{2}}{2 d}\right)\left(\left(\frac{W}{P}\right)^{2}+\delta \frac{P}{W}\right) d v_{x}
$$

where $\Delta f$ is the frequency range, $\delta$ is the noise temperature parameter, and $T_{0}$ is the lattice temperature.

The mean square drain noise voltage is given as

$$
\begin{aligned}
\overline{v_{d 1}^{2}}= & \frac{16 k T \Delta f K_{4}^{2}\left|V_{\text {off }}\right|}{q z v_{\text {sat }}\left(\sqrt{P_{1}}-K_{2}\right)^{2}} \cosh ^{2}\left(\frac{\pi L_{2}}{2 d}\right) \\
& \times\left(\left(\frac{1}{P^{2}}\right)\left(\frac{s^{3}-p^{3}}{3}\right)+\delta p \ln \left(\frac{S}{P}\right)\right) .
\end{aligned}
$$

Open circuit drain voltage fluctuation due to diffusion noise can be given as

$$
\begin{aligned}
\overline{v_{d 2}^{2}}= & \frac{64 q D \Delta f I_{d}(d+b)^{3}}{\pi^{5} \varepsilon^{5} z^{2} v_{\mathrm{sat}}^{3} b^{5}} \sin ^{2}\left(\frac{\pi b}{2(d+b)}\right) \\
& \times\left(3+\exp \left(\frac{\pi L_{2}}{d+b}\right)-4 \exp \left(\frac{\pi L_{2}}{2(d+b)}\right)+\frac{\pi L_{2}}{(d+b)}\right),
\end{aligned}
$$

where $b$ is the effective channel thickness and is given as

$$
b=\frac{\varepsilon d}{q d Q}\left\{E f\left(L_{1}\right)\right\}=\frac{\varepsilon}{q}\left(\frac{K_{2} K_{4}}{\sqrt{P_{1}}-K_{2}}+K_{3}\right) .
$$

5.2. Gate Circuit Noise. The noise current flowing may be obtained by $\overline{\Delta l_{g 2}^{2}}=\overline{\Delta v_{d 2}^{2}} / r_{d}^{2}$, where $r_{d}$ is the drain resistance.

The expression for short circuit gate noise current can be obtained as

$$
\begin{aligned}
& \overline{\Delta l_{g 2}^{2}} \\
& =\left(\frac{16 q^{3} \Delta f w^{2}(d+b)^{3} D L_{1}^{2} k_{0}^{2}}{\pi^{5} \varepsilon^{2} b^{2} v_{\text {sat }}^{3} k_{4}^{4} r_{d}^{2}}\right) \sin ^{2}\left(\frac{\pi b}{2(d+b)}\right) \\
& \quad \times\left\{3+\exp \left(\frac{\pi L_{2}}{(d+b)}\right)-4 \exp \left(\frac{\pi L_{2}}{2(d+b)}\right)+\left(\frac{\pi L_{2}}{(d+b)}\right)\right\},
\end{aligned}
$$

where

$$
K_{0}=K+\frac{L_{2}}{2 L_{1}}\left(\sqrt{P_{1}}-K_{2}\right)^{2}
$$

5.3. Noise Coefficients. The correlation between the drain noise source and the gate noise source is given as

$$
\begin{aligned}
& \overline{L_{g_{1}} * L_{d_{1}}} \\
& =\frac{-j w^{2} k T_{0} \Delta f q z\left|V_{\text {off }}\right| L_{1}}{K_{4}^{2} I_{d}^{2} r_{d}^{2}} \cosh \left(\frac{\pi L_{2}}{2 d}\right)\left(s_{0}+s_{1}+s_{2}+s_{3}\right) .
\end{aligned}
$$

The details of (39) are reported in [16].

The drain noise coefficient associated with the drain noise current is given as

$$
\begin{aligned}
P & =\frac{\overline{V_{d}^{2}}}{4 K T_{0} \Delta f g_{m}}=\frac{\overline{V_{d}^{2}}}{4 K T_{0} \Delta f g_{m} r_{d}^{2}} \\
& =\frac{\overline{l d_{1}^{2}}+\overline{l d_{2}^{2}}}{4 K T_{0} \Delta f g_{m}}=\frac{\overline{l d_{1}^{2}}+\overline{l d_{2}^{2}}}{4 K T_{0} \Delta f g_{m} r_{d}^{2}}=P_{1}+P_{2} .
\end{aligned}
$$

The gate noise coefficient associated with the gate noise current is given as

$$
R=\frac{\overline{l_{g}^{2}} g_{m}}{4 K T_{0} \Delta f w^{2} C_{\mathrm{gs}}^{2}}=\frac{\left(\overline{l_{g_{1}}^{2}}+\overline{l_{g_{2}}^{2}}\right) g_{m}}{4 K T_{0} \Delta f w^{2} C_{\mathrm{gs}}^{2}}=R_{1}+R_{2},
$$

where $\overline{V_{d}^{2}}$ is the equivalent noise voltage at the drain region and $\overline{i_{g}^{2}}$ is the equivalent noise induced gate current.

The noise coefficients $P_{1}$ and $P_{2}$ and $R_{1}$ and $R_{2}$ are of the same form as $P$ and $R$, but with subscripts 1 and 2 on different noise sources in the ohmic and saturation region, respectively.

The correlation coefficient between different noise sources can be written as

$$
C=\frac{\overline{l_{g} * l_{d}}}{\sqrt[4]{l_{g}^{2} l_{d}^{2}}}=C_{1}+C_{1} .
$$

The correlation coefficient in the linear region can be given in terms of $P$ and $R$ as

$$
C_{1}=C_{11} \cdot \sqrt{\frac{P_{1} R_{1}}{P R}}
$$


The correlation coefficient in the saturation region can be given in terms of $P$ and $R$ as

$$
C_{2}=C_{22} \cdot \sqrt{\frac{P_{2} R_{2}}{P R}}
$$

The correlation coefficient between $l_{g_{1}}$ and $l_{d_{1}}$ can be calculated as

$$
C_{11}=\frac{\sqrt{l_{g_{1}} * l_{d_{1}}}}{\sqrt[5]{l_{g_{1}}^{2} l_{d_{1}}^{2}}}
$$

There is full correlation between $i_{d_{2}}$ and $i_{g_{2}}$ with a capacitive phase shift of $90^{\circ}$. Therefore,

$$
C_{22}=\frac{\overline{l_{g_{2}} * l_{d_{2}}}}{\sqrt[5]{l_{g_{2}}^{2} l_{d_{2}}^{2}}}=1
$$

Therefore, there is no correlation between $i_{d_{1}}$ and $i_{g_{2}}$ or $i_{d_{2}}$ and $i_{g_{1}}$.

5.4. Noise Figure. The minimum noise figure in terms of source resistance and gate resistance can be calculated as

$$
F_{\min }=1+2 g_{n}\left(R_{c}+\sqrt{R_{c}^{2}+\frac{r_{n}}{g_{n}}}\right),
$$

where

$$
\begin{aligned}
g_{n}= & \text { noise conductance }=\frac{w^{2} C_{\mathrm{gs}}^{2}}{g_{m}}(P+R-2 C \sqrt{P R}) \\
= & K_{g} \frac{w^{2} C_{\mathrm{gs}}^{2}}{g_{m}}, \\
r_{n} & =\text { noise resistance } \\
& =R_{s}+R_{g}+\frac{1+w^{2} C_{\mathrm{gs}}^{2} R_{i}^{2}}{g_{m}} \cdot \frac{P R\left(1-C^{2}\right)}{P+R-2 C \sqrt{P R}} \\
& R_{s}+R_{g}+K_{r}\left(\frac{1+w^{2} C_{\mathrm{gs}}^{2} R_{i}^{2}}{g_{m}}\right), \\
& =R_{s}+R_{g}+\left(R_{i}-\frac{j}{w C_{\mathrm{gs}}}\right) \cdot \frac{P-C \sqrt{P R}}{P+R-2 C \sqrt{P R}} \\
& =R_{s}+R_{g}+K_{c}\left(R_{i}-\frac{j}{w C_{\mathrm{gs}}}\right),
\end{aligned}
$$

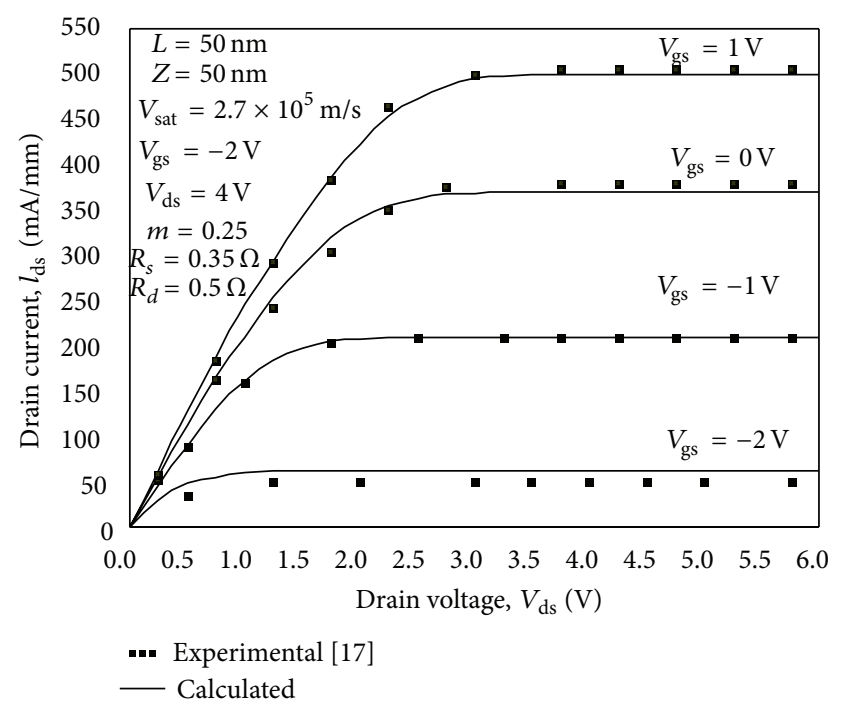

FIGURE 2: I-V characteristics of AlGaN/GaN MODFET.

where $K_{g}, K_{r}$, and $K_{c}$ are the functions of $P, R$, and $C$ and are given as

$$
\begin{aligned}
& K_{g}=P+R-2 C \sqrt{P R}, \\
& K_{r}=\frac{P R\left(1-C^{2}\right)}{P+R-2 C \sqrt{P R}}, \\
& K_{c}=\frac{P-C \sqrt{P R}}{P+R-2 C \sqrt{P R}} .
\end{aligned}
$$

5.5. Noise Temperature. The minimum noise temperature can be obtained as

$$
T_{\min }=2 T g_{n}\left(R_{c}+Z_{c}\right)
$$

where $T$ is absolute temperature and $R_{c}$ is the correlation resistance.

\section{Results and Discussion}

To prove the validity of the model, the calculated/obtained results are plotted and compared with the previously published experimental and simulated results.

Figure 2 shows the current-voltage characteristics of AlGaN/GaN MODFET of $50 \mathrm{~nm}$ gate length for different values of gate voltages. It can be seen that current increases with the increase in drain-source voltage. A peak drain current of $501 \mathrm{~mA} / \mathrm{mm}$ is achieved for gate bias of $1 \mathrm{~V}$. The model exhibits high current driving capabilities and good pinch-off characteristics. In saturation region, the current continues to increase slightly due to short-channel effects. The calculated results are in good agreement with the experimental results [17]. 


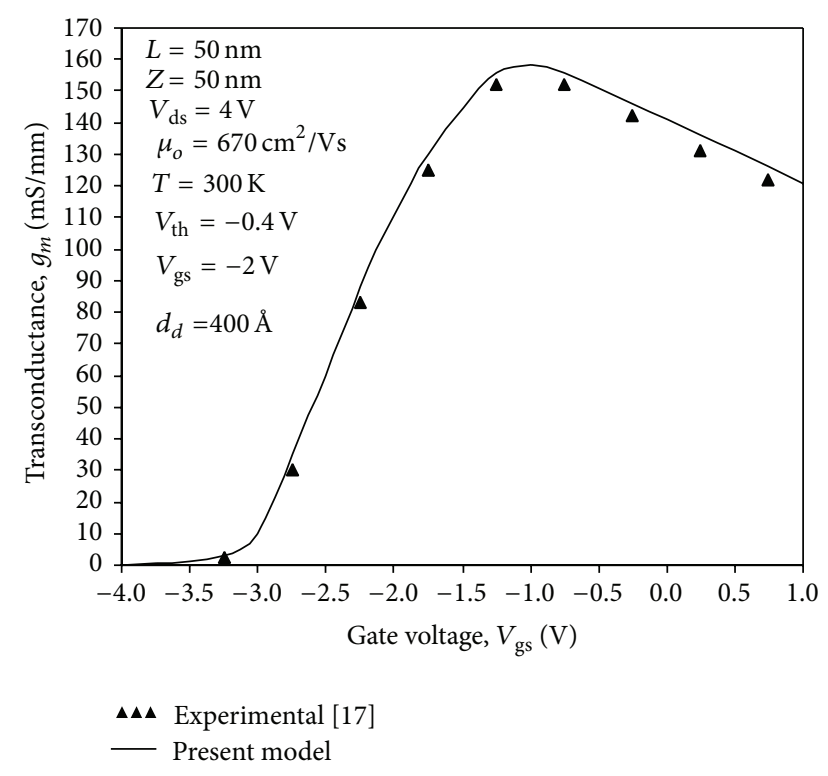

FIGURE 3: Variation of transconductance with gate voltage.

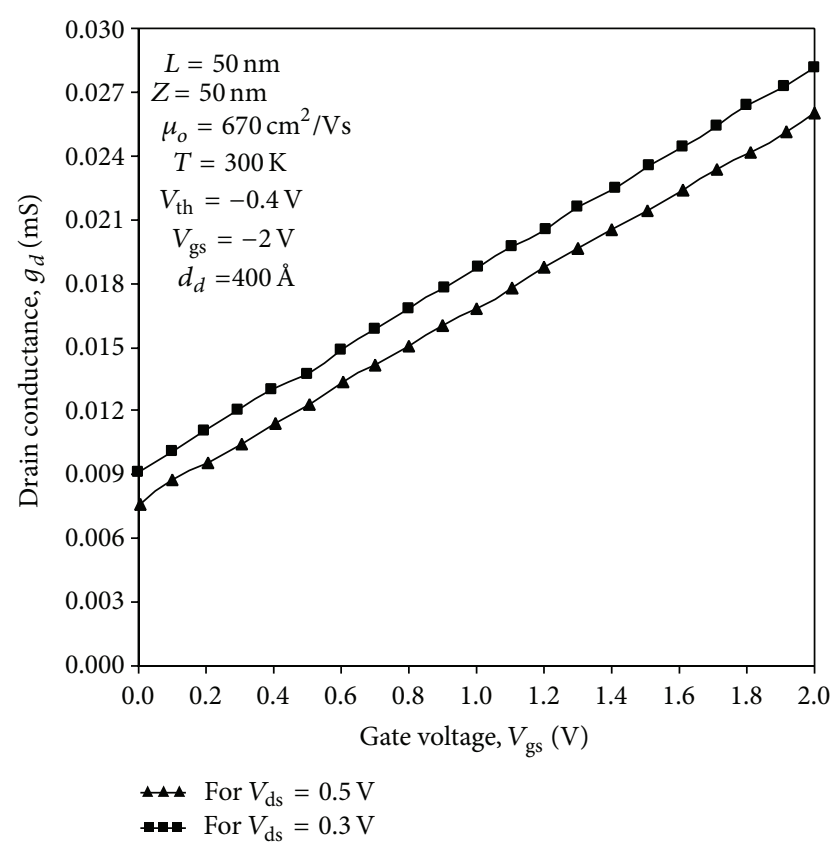

Figure 4: Variation of drain conductance with gate voltage.

The variation of transconductance with gate voltage is depicted in Figure 3. The high value of transconductance can be explained due to high saturation velocity and better carrier mobility. The calculated transconductance has a peak value of $160 \mathrm{mS} / \mathrm{mm}$ at a gate bias of $-1.2 \mathrm{~V}$. As $V_{\mathrm{gs}}$ increases, the pHEMT initially operates in the saturation region. As $V_{\mathrm{gs}}$ continues to increase, the transistor enters in the linear region

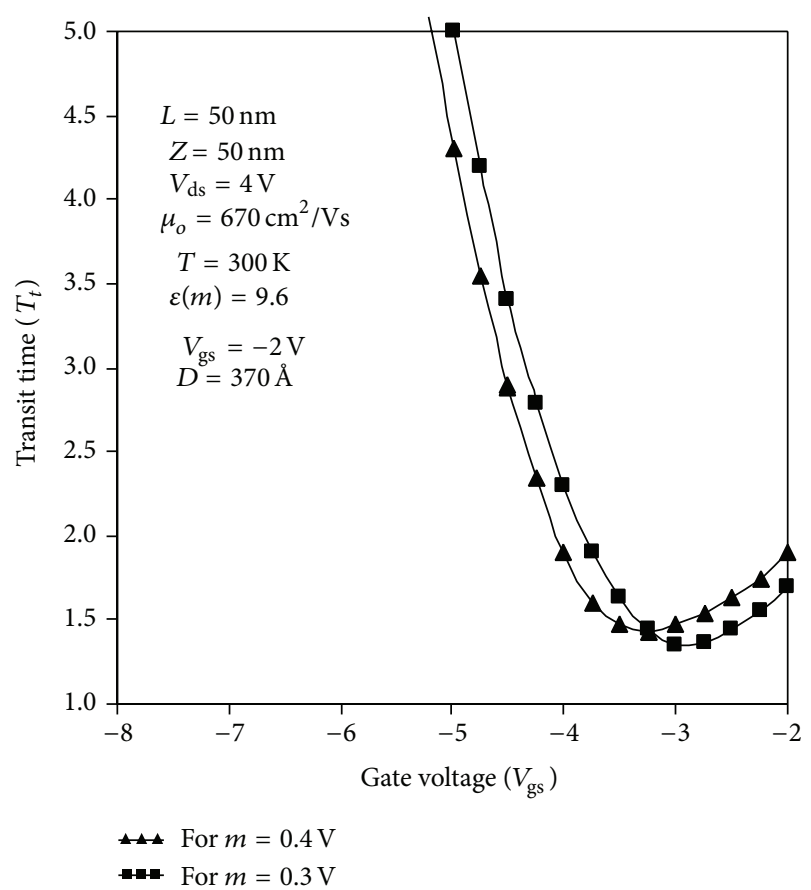

FIGURE 5: Variation of transit time with gate voltage for different mole fraction.

of operation and the transconductance decreases. The present results are in close agreement with the experimental results [17].

The plot of output/drain conductance for different gate voltages is shown in Figure 4. As shown in the figure, the output conductance increases gradually with the increases in gate voltage for different values of drain bias. Starting from left to right, the transistor starts in saturation and slowly approaches the linear region as gate bias increases.

The variation of transit time with gate voltage is depicted in Figure 5. The figure shows the effect of $\mathrm{Al}$ mole fraction composition on transit time variation with gate bias. Minimum value of transit time is achieved at the gate voltage corresponding to the peak transconductance. Increase in $\mathrm{Al}$ mole content shifts the gate voltage to a minimum transit time.

Figure 6 shows the variation of cut-off frequency with drain current. For low values of drain current, transconductance is low and hence cut-off frequency is also low. At a drain current of $203 \mathrm{~mA} / \mathrm{mm}$, a cut-off frequency of $11.6 \mathrm{GHz}$ is obtained. The present results are in close proximity with the experimental results [18].

Figure 7 shows the dependence of gate-source capacitance on drain-source voltage. It is observed from the figure that, for small drain voltages, the dependence is small and for higher values of drain voltage there is rapid rise in gate-source capacitance due to the carrier saturation in the device.

Figure 8 shows the variation of $C_{\mathrm{gs}}$ with $V_{\mathrm{gs}}$ for different values of $V_{\mathrm{ds}}$. It is evident from the figure that $C_{\mathrm{gs}}$ decreases with decrease in $V_{\mathrm{gs}}$ and increases with increase in $V_{\mathrm{gs}}$. 


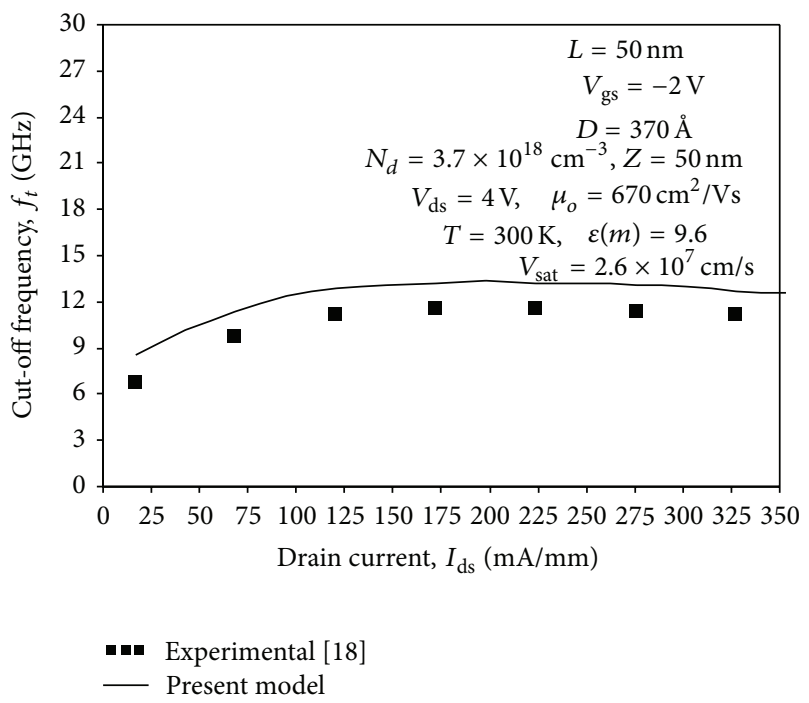

FIGURE 6: Variation of cut-off frequency with drain current.

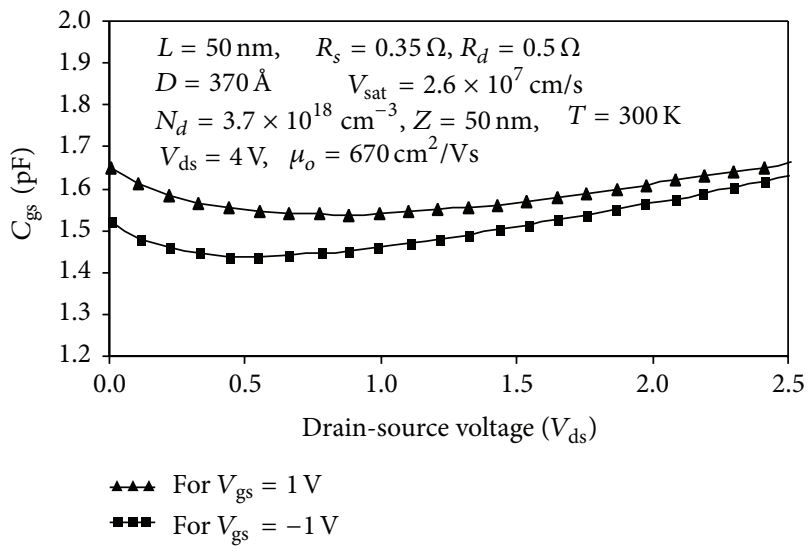

Figure 7: Dependence of $C_{\mathrm{gs}}$ on $V_{\mathrm{ds}}$.

Figure 9 shows the variation of $C_{\mathrm{gd}}$ with $V_{\mathrm{dg}}$ for different values of $V_{\mathrm{gs}}$. It can be seen from the figure that there is sharp increase in $C_{\mathrm{gd}}$ at $V_{\mathrm{dg}}$ of $-3 \mathrm{~V}$. The calculated results are in good agreement with experimental results [13].

The variation of PAE, gain, and output power with input power is shown in Figure 10. A PAE of 54\% with an associated power gain of $14 \mathrm{~dB}$ and $31 \mathrm{dBm}$ output power with a frequency of $20 \mathrm{GHz}$ is obtained. It is evident from the figure that the input power varies linearly with output power. These plots are consistent with those reported in [19].

Figures 11, 12, and 13 show the dependence of noise coefficients $(P, R$, and $C)$ on drain current. The correlation coefficient $C$ shows the symmetric behaviour. It is almost constant for all values of drain currents. It decreases with shorter gate lengths. The gate noise coefficient $R$ is very

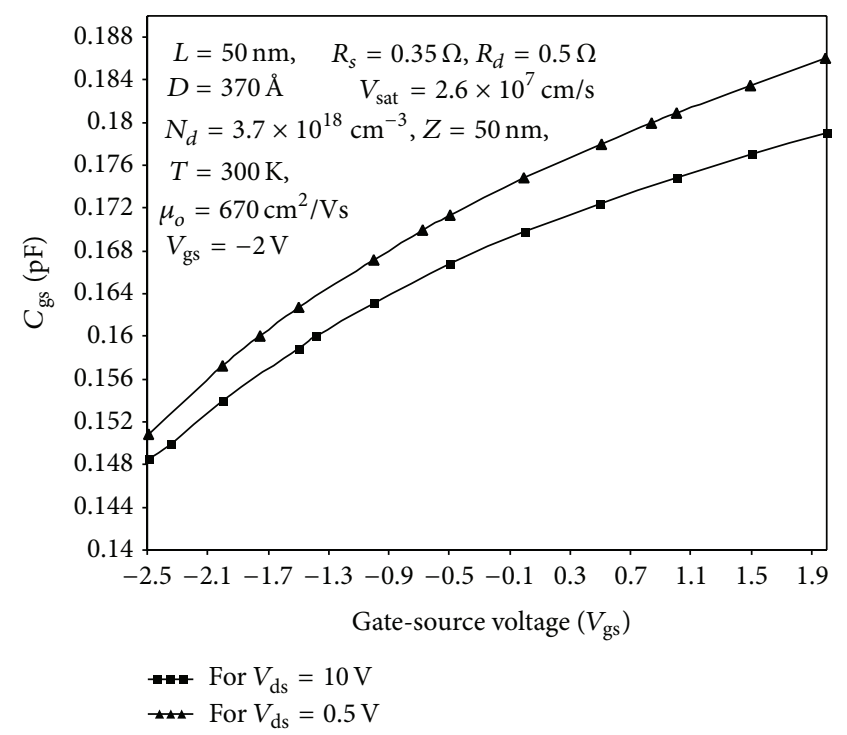

FIgURE 8: Variation of $C_{\mathrm{gs}}$ with gate voltage.

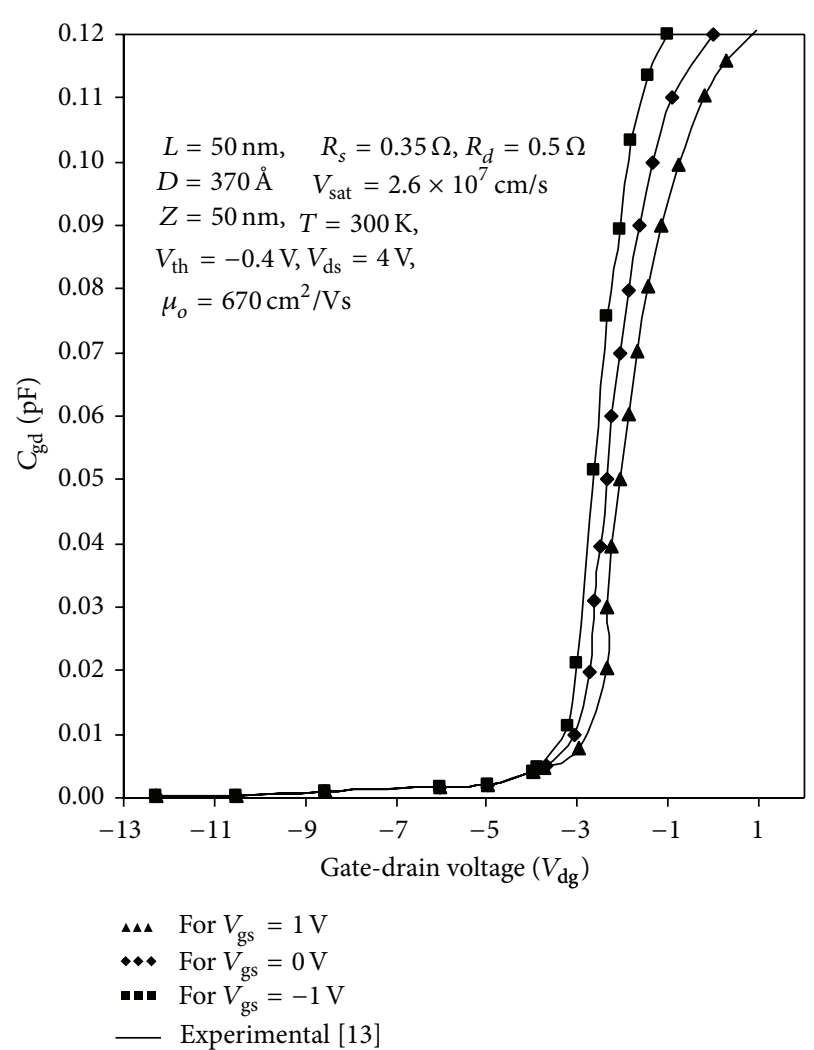

Figure 9: Variation of $C_{\mathrm{gd}}$ with $V_{\mathrm{dg}}$.

high at low drain currents due to decrease in gate to source capacitance. It increases for shorter gate lengths. The drain noise coefficient $P$ decreases with lower values of drain currents. It also decreases for shorter gate lengths. The calculated/obtained results are in close proximity with the previously published results [20]. 


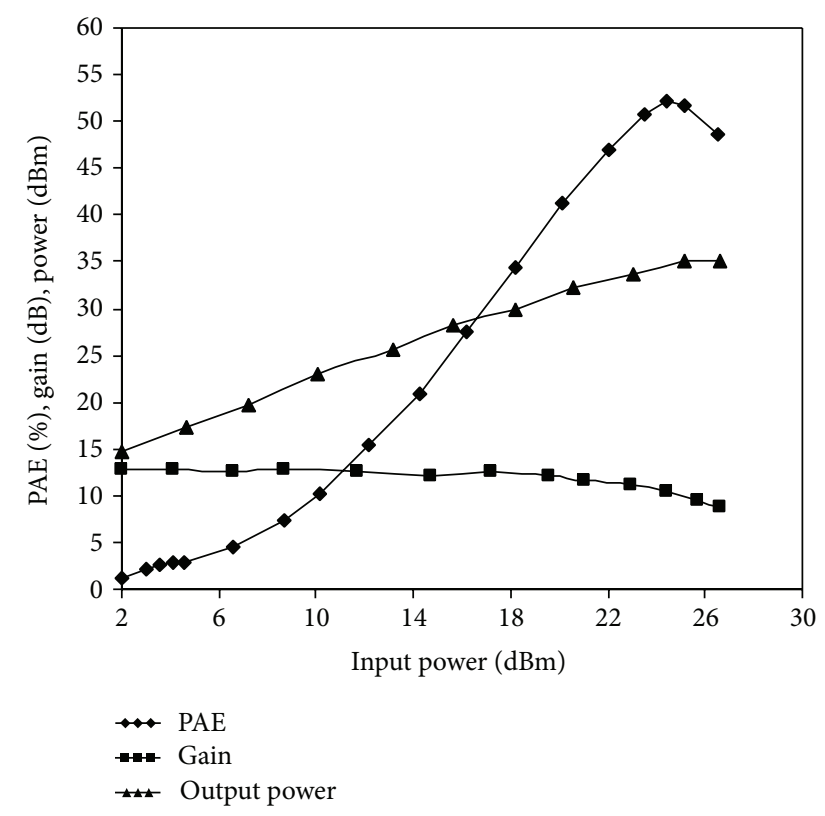

Figure 10: Variation of PAE, gain, and output power with input power.

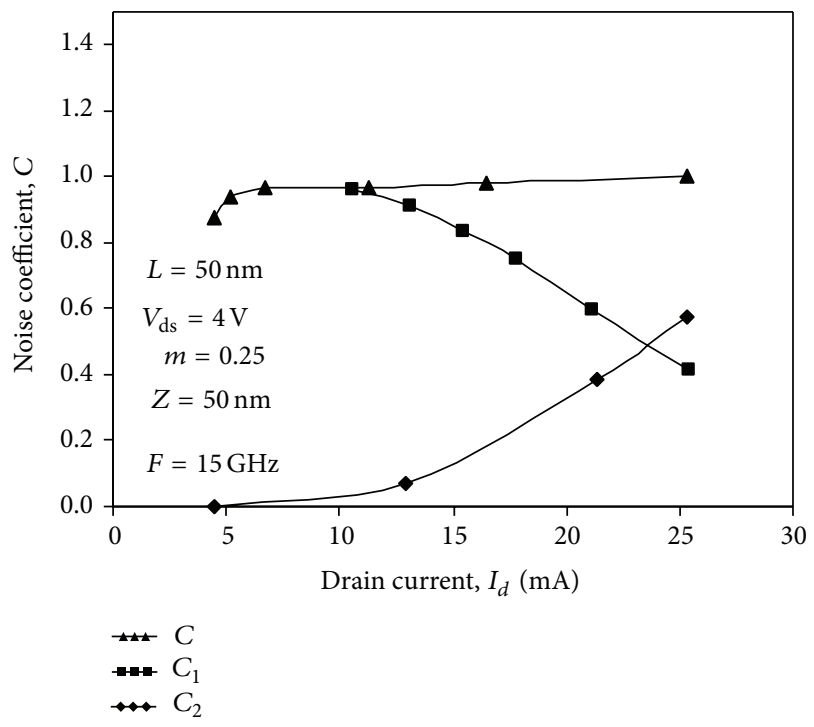

Figure 11: Dependence of noise correlation coefficient $C$ on drain current.

Figure 14 shows the dependence of noise temperature on drain current for $50 \mathrm{~nm}$ gate length which is consistent with those reported in [20]. It can be seen from the graph that the transistor achieves lower noise temperature due to better confinement and effective channel width.

The variation of minimum noise figure with frequency is presented in Figure 15. It is clear from the figure that, with increase in frequency, minimum noise figure also increases. The results are in close agreement with the previously published results [20].

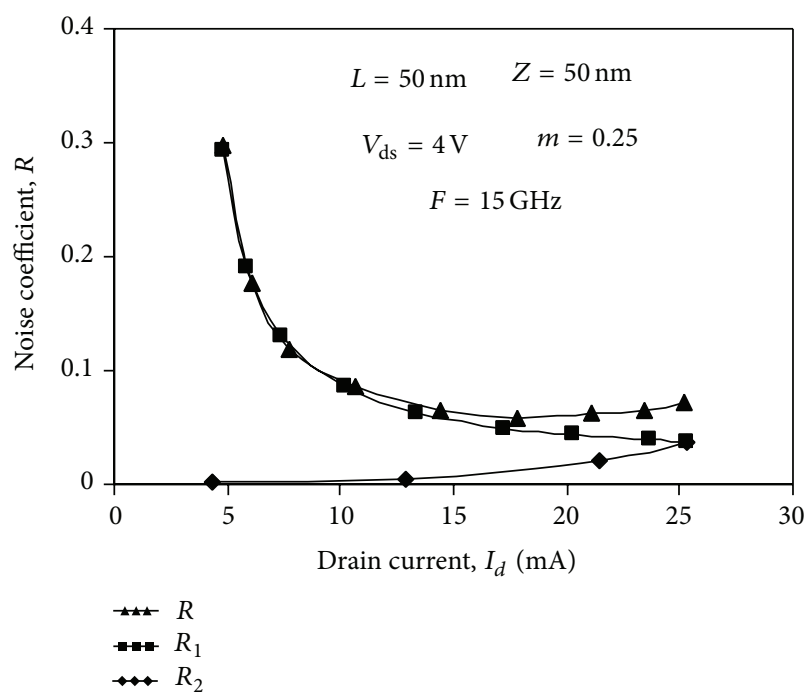

FIGURE 12: Variation of gate noise coefficient $R$ with drain current.

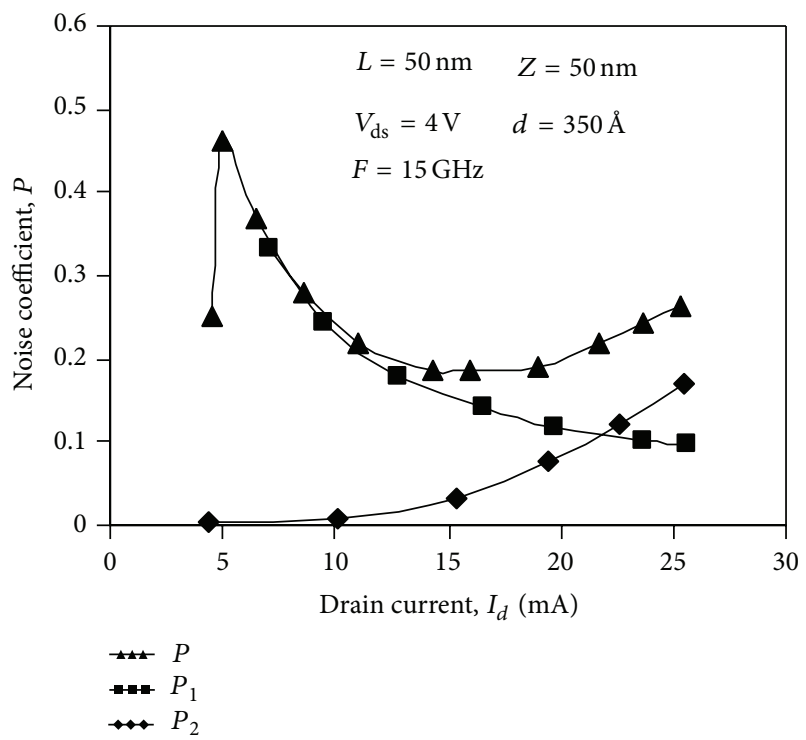

FIGURE 13: Variation of drain noise coefficient $P$ with drain current.

\section{Conclusion}

The proposed model is developed for the 2D MODFET in characterizing and evaluating the $\mathrm{dc}$ and microwave performance for a gate length of $50 \mathrm{~nm}$ device. The model is developed for the I-V characteristics and small signal parameters of an AlGaN/GaN MODFET considering the strong polarization effects. Including the analysis of the physical meaning of the capacitances, the $\mathrm{C}-\mathrm{V}$ model reflects the variations in the capacitance with $V_{\mathrm{gs}}$ and $V_{\mathrm{ds}}$. The dependencies of noise performance on drain current and frequency were characterized. The noise analysis is quiet good in linear and saturation region. All the noise coefficients like drain noise coefficient $(P)$, gate noise coefficient $(R)$, and 


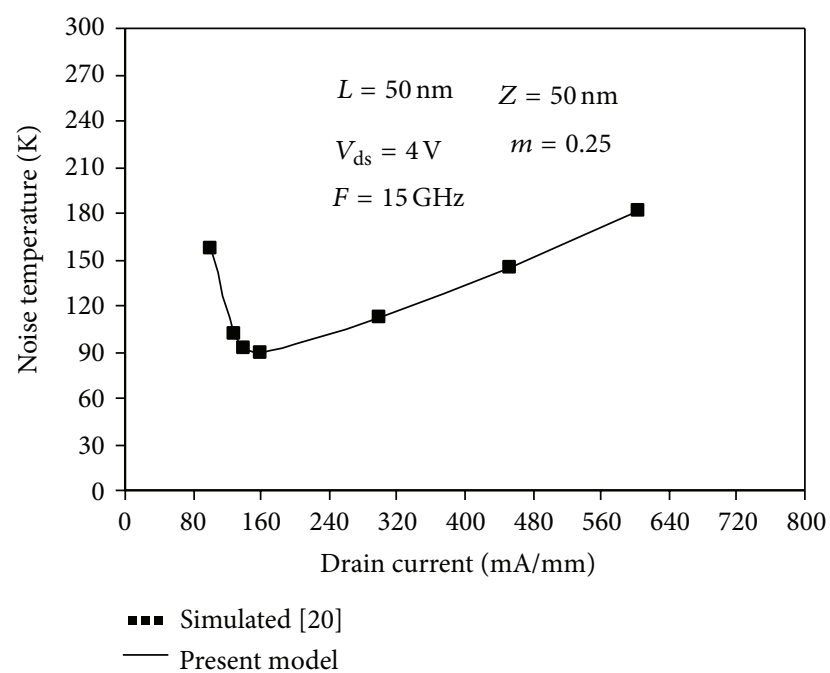

FIGURE 14: Variation of noise temperature with drain current.

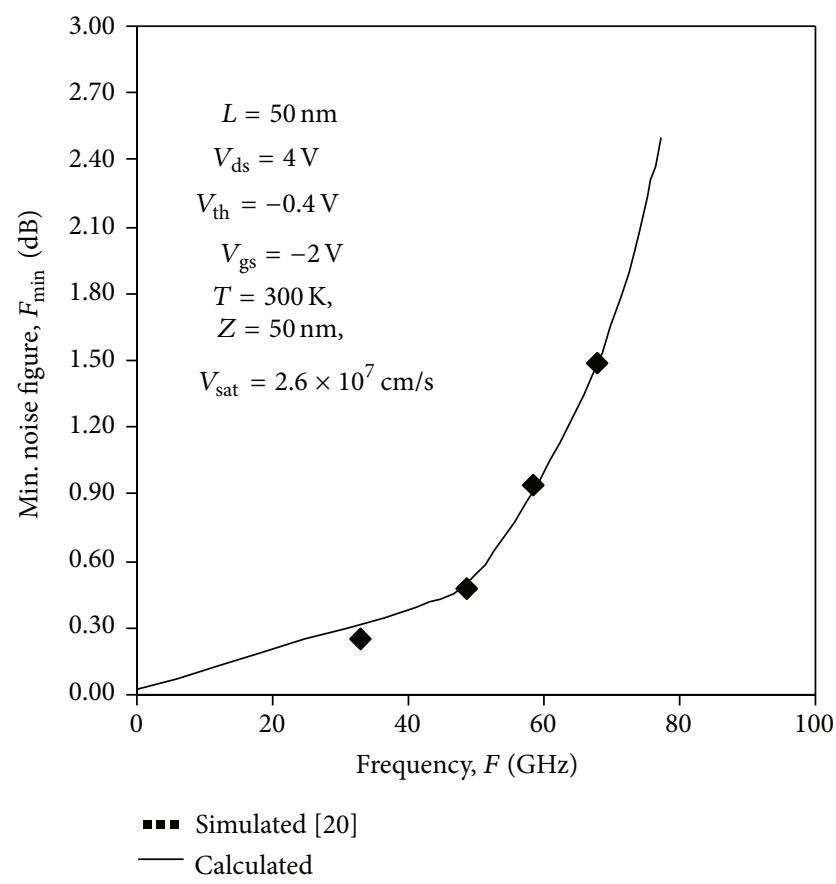

FIGURE 15: Dependence of minimum noise figure on frequency.

correlation coefficient $(C)$ and noise figure are also evaluated. The comparisons between the power performance and the model simulation validate the approximations of the present capacitance and noise model.

\section{Conflict of Interests}

The authors declare that there is no conflict of interests regarding the publication of this paper.

\section{References}

[1] M. Bhattacharya, J. Jogi, R. S. Gupta, and M. Gupta, "Scattering parameter based modeling and simulation of symmetric tiedgate InAlAs/InGaAs DG-HEMT for millimeter-wave applications," Solid-State Electronics, vol. 63, no. 1, pp. 149-153, 2011.

[2] A. Ahlawat, M. Pandey, and S. Pandey, "Microwave analysis of A $70 \mathrm{~nm}$ InGaAs pHEMT on InP substrate for nanoscale digital IC application," Microwave and Optical Technology Letters, vol. 49, no. 10, pp. 2462-2470, 2007.

[3] Rashmi, A. Kranti, S. Haldar, and R. S. Gupta, "An accurate charge control model for spontaneous and piezoelectric polarization dependent two-dimensional electron gas sheet charge density of lattice-mismatched AlGaN/GaN HEMTs," Solid-State Electronics, vol. 46, no. 5, pp. 621-630, 2002.

[4] O. Ambacher, J. Smart, J. R. Shealy et al., "Two-dimensional electron gases induced by spontaneous and piezoelectric polarization charges in N- And Ga-face AIGaN/GaN heterostructures," Journal of Applied Physics, vol. 85, no. 6, pp. 3222-3233, 1999.

[5] R. K. Tyagi, A. Ahlawat, M. Pandey, and S. Pandey, "An analytical two-dimensional model for AlGaN/GaN HEMT with polarization effects for high power applications," Microelectronics Journal, vol. 38, no. 8-9, pp. 877-883, 2007.

[6] R. Kumar, S. K. Arya, and A. Ahlawat, "Analysis of smallsignal parameters of 2-D MODFET with polarization effects for microwave analysis," International Journal of VLSI Design \& Communication, vol. 4, no. 2, pp. 51-61, 2013.

[7] R. A. Pucel, H. A. Haus, and H. Statz, "Signal and noise properties of gallium arsenide microwave field effect transistors," Advances in Electronics and Electron Physics, vol. 38, pp. 195265, 1975.

[8] T. M. Brookes, "The noise properties of high electron mobility transistors," IEEE Transactions on Electron Devices, vol. 33, no. 1, pp. 52-57, 1986.

[9] V. Kumar, W. Lu, R. Schwindt et al., "AlGaN/GaN HEMTs on $\mathrm{SiC}$ with $f_{T}$ of over $120 \mathrm{GHz}$," IEEE Electron Device Letters, vol. 23, no. 8, pp. 455-457, 2002.

[10] S. Sen, M. K. Pandey, and R. S. Gupta, “Two-dimensional C-V model of AlGaAs/GaAs modulation doped field effect transistor (MODFET) for high frequency applications," IEEE Transactions on Electron Devices, vol. 46, no. 9, pp. 1818-1823, 1999.

[11] H. K. Huang, Y. H. Wang, C. L. Wu, J. C. Wang, and C. S. Chang, "Super low noise InGaP gated PHEMT," IEEE Electron Device Letters, vol. 23, no. 2, pp. 70-72, 2002.

[12] F. E. Rangel Patino and J. R. Camacho Perez, "Modeling and simulation of pseudomorphic HEMTs for analog circuit design and analysis," in Delphi Automotive Systems/Delco Electronics, pp. 277-282, Instituto Tecnologico de Chihuahua, Electro, 2001.

[13] J. C. Sippel, S. S. Islam, and S. S. Mukherjee, "A physics-based model of DC and microwave characteristics of GaN/AlGaN HEMTs," International Journal of RF and Microwave ComputerAided Engineering, vol. 17, no. 3, pp. 254-264, 2007.

[14] L. Stepan, "Power-added efficiency errors with RF power amplifiers," International Journal of Electronics, vol. 82, no. 3, pp. 303-312, 1997.

[15] P. Yan, W. Liang, Y. Tingting et al., "Multi-bias capacitance voltage characteristic of AlGaN/GaN HEMT," Journal of Semiconductors, vol. 31, no. 10, Article ID 104002, 2010.

[16] M. Bhattacharya, J. Jogi, R. S. Gupta, and M. Gupta, "An accurate charge-control-based approach for noise performance assessment of a symmetric tied-gate InAlAs/InGaAs DG-HEMT," 
IEEE Transactions on Electron Devices, vol. 59, no. 6, pp. 16441652, 2012.

[17] R. Angu Agrawal, S. Sen, S. Haldar, and R. S. Gupta, "Analytical model for dc characteristics and small signal parameters of AlGaN / GaN modulation-doped field effect transistor for microwave circuit applications," Microwave and Optical Technology Letters, vol. 27, no. 6, pp. 413-419, 2000.

[18] Y.-F. Wu, S. Keller, P. Kozodoy et al., "Bias dependent microwave performance of AlGaN/GaN MODFET's up to $100 \mathrm{~V}$,' IEEE Electron Device Letters, vol. 18, no. 6, pp. 290-292, 1997.

[19] C. Campbell, C. Lee, V. Williams et al., "A wideband power amplifier MMIC utilizing GaN on SiC HEMT technology," IEEE Journal of Solid-State Circuits, vol. 44, no. 10, pp. 2640-2647, 2009.

[20] R. K. Tyagi, A. Ahlawat, M. Pandey, and S. Pandey, "Noise analysis of sub quarter micrometer AlGaN/GaN microwave power HEMT," Journal of Semiconductor Technology and Science, vol. 9, no. 3, pp. 125-135, 2009. 

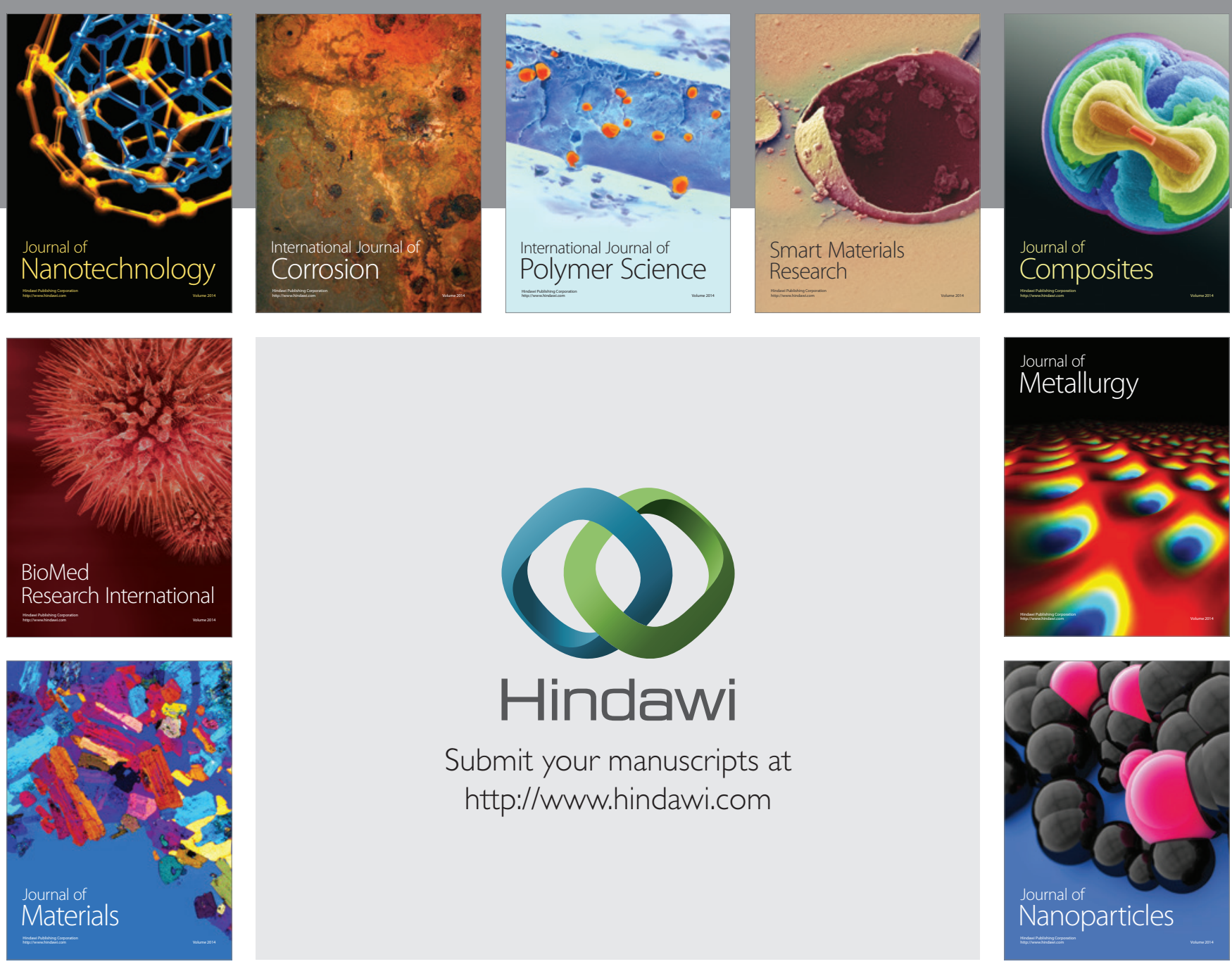

Submit your manuscripts at http://www.hindawi.com
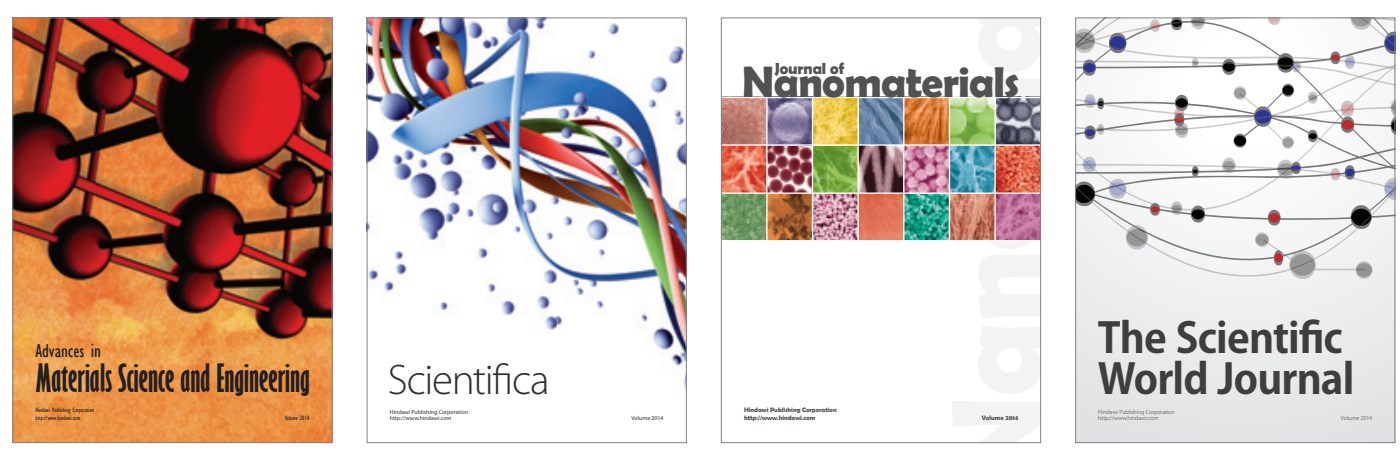

\section{The Scientific World Journal}
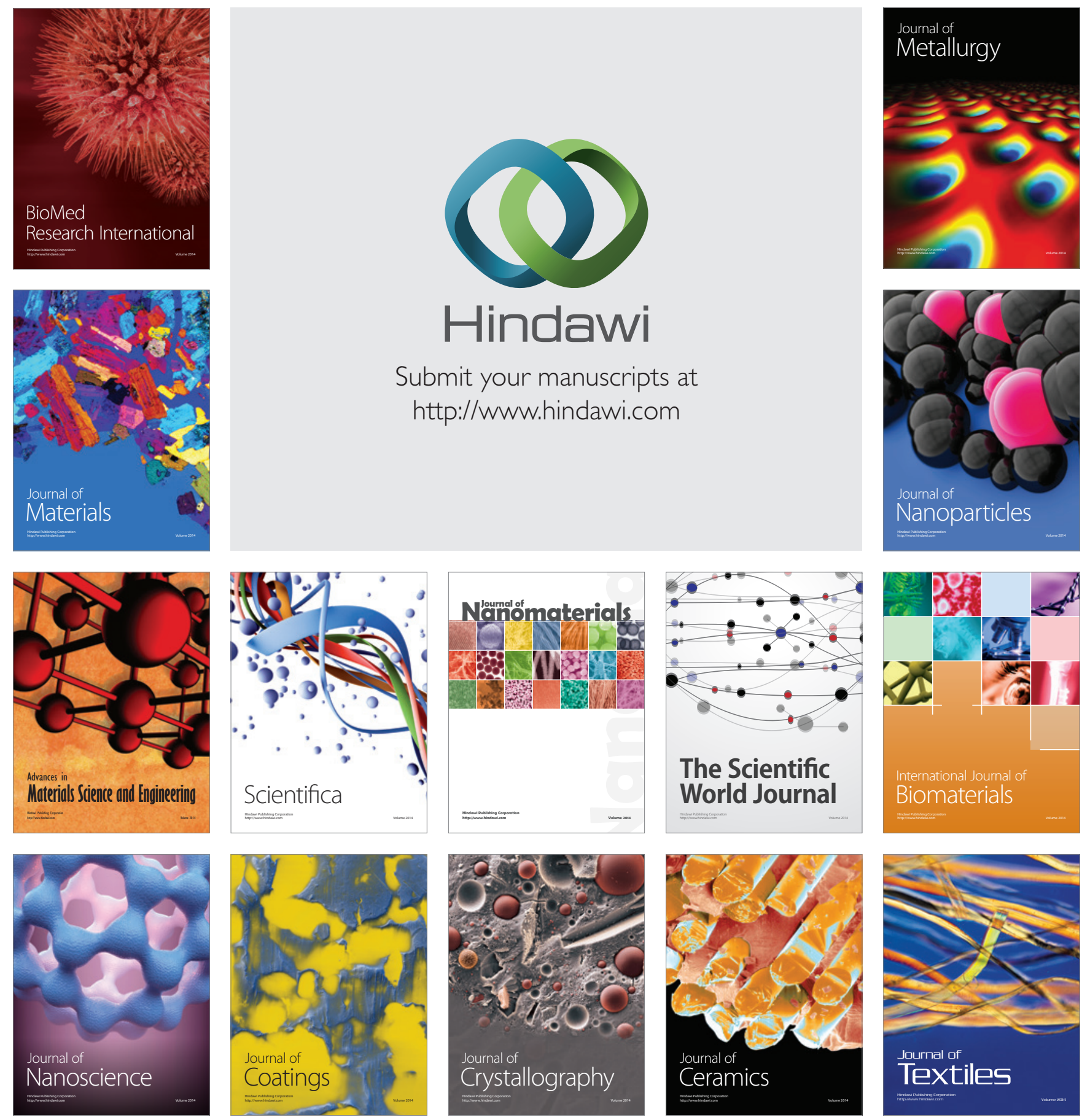